Molecular Physics, 1982, Vol. 45, No. 2, 427-439

\title{
Calculation of transition metal compounds using an extension of the CNDO formalism
IV. CNDO-CI-calculations on $\mathrm{Ni}(\mathrm{CO})_{4}$ and $\mathrm{Fe}(\mathrm{CO})_{5}$; electronic spectra and photochemical implications $\dagger$

\author{
by BERNHARD DICK, HANS-JOACHIM FREUND \\ and GEORG HOHLNEICHER \\ Lehrstuhl für Theoretische Chemie der Universität zu Köln, \\ 5000 Köln 41, Greinstraße 4, West Germany
}

(Received 13 October 1980 ; accepted 25 September 1981)

\begin{abstract}
CI-calculations based on an extended CNDO formalism, introduced in Part I of this series have been used to analyse the low lying excited states of $\mathrm{Ni}(\mathrm{CO})_{4}$ and $\mathrm{Fe}(\mathrm{CO})_{5}$. In contrast to the usual assumption, that, apart from $d \rightarrow d$ excitations, the carbonyl U.V. spectra are determined exclusively by charge-transfer transitions, atomic $d \rightarrow s$ and $d \rightarrow p$ excitations are found to be important in the low energy region. Inspection of the density matrices of excited states indicates that these transitions are responsible for the photochemical activity of metal carbonyls. The influence of doubly excited configurations is reduced with respect to what is found for organic compounds.
\end{abstract}

\section{Introduction}

The electronic spectra of simple carbonyls are not well understood yet. The relatively strong ligand metal interaction prevents a satisfactory description of the low lying excitation in terms of the metal electrons alone. Extended Hückel-type methods [1] and $\mathrm{X}_{\alpha}-\mathrm{SW}$ treatments [2] have been applied to such systems. Within both approaches the energy of U.V. absorptions are directly associated with orbital energy differences. The orbital degeneracy does, however, require a configuration interaction treatment (CI) to achieve an even qualitatively correct assignment of spectral transitions. We have used an extended CNDO-formalism previously introduced $[3,4]$ including CI to study the electronic spectra of simple carbonyls. The method was especially developed for the treatment of systems containing transition metal atoms, and we present, as first examples, the results for $\mathrm{Ni}(\mathrm{CO})_{4}$ and $\mathrm{Fe}(\mathrm{CO})_{5}$. Together with excitation energies we calculate oscillator strengths and two-photon transition probabilities. The charge and bond order matrices of the low-lying excited states are analysed to provide information on the strong photochemical activity of the simple carbonyls.

\section{Method of Calculation}

The SCF ground states are calculated by an extended CNDO-type method described in detail in $[3(a)]$. Correlated wavefunctions for ground and excited

† For Part I-V see [3].

0026-8976/82/4502 0427 \$04.00 1982 Taylor \& Francis Ltd 
states are calculated by a CI which includes, to some extent, doubly excited configurations (DEC) [5], since we know from recent results on organic compounds [5-7], that DECs may strongly influence the ordering of low lying states. Within the semiempirical methods the effect depends on the mathematical form used to represent the Coulomb integrals as a function of internuclear distance. The steeper this function, the more important are the DECs [5]. In our CNDO procedure the integrals are calculated using Slater orbitals leading to a steeper distance dependence than any of the usually applied semiempirical formulas. We have therefore to expect an overestimation of the influence of DECs rather than an underestimation.

It is known that SCF-CI calculations. on organic molecules often lead to unsatisfactory results for electronic excitation energies if the standard CNDO/2 parametrization $[8,9]$ is used. This is due mainly to an overestimation of $\pi$-type interactions. Fortunately this is not the case for the $\mathrm{CO}$ molecule; the first three excitations obtained by $\mathrm{CNDO} / 2$ are in qualitative agreement with $a b$ initio results [10] (compare table 1). Two CNDO-CI calculations have been performed for $\mathrm{CO}$; one includes all singly and doubly excited configurations (SDCI (full)) and the second only those with energies lower than one atomic unit (SDCI (34)). From a comparison of both calculations we see that it is mainly the ground state which is stabilized by inclusion of high energy DECs. The energy difference between excited states are only affected by less than $0.2 \mathrm{eV} . \mathrm{CO}$ is therefore one of the unusual cases where a $\mathrm{CNDO} / 2-\mathrm{CI}$ calculation including singly and doubly excited configurations with energies up to one atomic unit provides a reasonable basis to describe the first few excitations.

Table 1. Theoretical and experimental excitation energies (in $\mathrm{eV}$ ) for carbon monoxide. Oscillator strengths are given in brackets.

\begin{tabular}{|c|c|c|c|c|c|c|}
\hline \multicolumn{3}{|c|}{ State } & \multirow{2}{*}{$\frac{A b \text { initio } \dagger}{8.62(0.209)}$} & \multirow{2}{*}{$\frac{\operatorname{SDCI}(34)}{7.66(0.148)}$} & \multirow{2}{*}{$\frac{\text { SDCI (full) }}{9.19(0.122)}$} & \multirow{2}{*}{$\frac{\text { Experiment }}{8.39(0.195) t}$} \\
\hline$\tilde{A}$ & ${ }^{1} \Pi$ & $n \rightarrow \pi^{*}$ & & & & \\
\hline$\tilde{I}$ & ${ }^{1} \Sigma^{-}$ & $\pi \rightarrow \pi^{*}$ & $9 \cdot 52$ & $11 \cdot 29$ & 12.63 & $9.90 \S$ \\
\hline$\tilde{D}$ & ${ }^{1} \Delta$ & $\pi \rightarrow \pi^{*}$ & 10.04 & $11 \cdot 20$ & $12 \cdot 63$ & $10.50 \S$ \\
\hline$\widetilde{B}$ & $1 \Sigma^{+}$ & $n \rightarrow R$ & $11.95(0.041)$ & & & $10.78 t$ \\
\hline$\tilde{C}$ & $1 \Sigma^{+}$ & $n \rightarrow R$ & $12.67(0.083)$ & & & $11.40 t$ \\
\hline \multirow[t]{3}{*}{$\tilde{E}$} & $11 I$ & $n \rightarrow R$ & $12.63(0.058)$ & & & $12 \cdot 36$ \\
\hline & $1 \Pi$ & $\sigma \rightarrow \pi^{*}$ & & $13 \cdot 74(0.360)$ & $15.04(0.493)$ & \\
\hline & ${ }^{1} \Sigma^{+}$ & $\pi \rightarrow \pi^{*}$ & & $15 \cdot 36(0.042)$ & $16.49(0.045)$ & \\
\hline & & & & $\begin{array}{l}\dagger \text { From [10]. } \\
\text { t From [24]. } \\
\S \text { From [25]. } \\
\| \text { From [26]. }\end{array}$ & & \\
\hline
\end{tabular}

The one electron transition moment for excitation from orbital $|i\rangle$ to orbital $|j\rangle$ is given by

$$
R_{i j}=\sum_{A} \sum_{\mu(A)} c_{i \mu} c_{j \mu} R_{\mu \mu}+\sum_{A} \sum_{\mu(A) \neq \nu(A)} c_{i \mu} c_{j \nu} R_{\mu \nu}+\sum_{A \neq B} \sum_{\mu(A)} \sum_{\nu(B)} c_{i \mu} c_{j \nu} R_{\mu \nu},
$$


where $\mu$ and $\nu$ represent atomic orbitals located at the atoms shown in parentheses. The implication of the ZDO approximation to the relative importance of the three terms in (1) to the calculation of oscillator strengths has been discussed in detail by Jaffé $e t$ al. [11]. We only want to point out that the $R_{\mu \nu}$ appearing in the third term of (1) are always neglected within the ZDO approximation. As pure CT-transitions only gain intensity from this third term, the calculated transition probability is always zero in CNDO and related methods. As a consequence metal to ligand CT-transitions in the carbonyls are calculated with far too low an intensity.

Two independent quantities can be calculated which describe a two-photon transition in a system of randomly oriented molecules [12]. Most commonly used are the two-photon cross section for two parallel polarized photons of same energy $\delta_{\uparrow \uparrow}$ and the two-photon polarization parameter $\Omega$ which serves as a symmetry indicator. Both parameters are calculated according to procedures given in [13].

\section{RESUlts AND Discussion}

\subsection{Excited states and U.V. spectrum of $\mathrm{Ni}(\mathrm{CO})_{4}$}

The calculated orbital energies of $\mathrm{Ni}(\mathrm{CO})_{4}$ are shown in figure 1 , together with the eigenvalue spectra of the pure ligand, the complete ligand sphere and

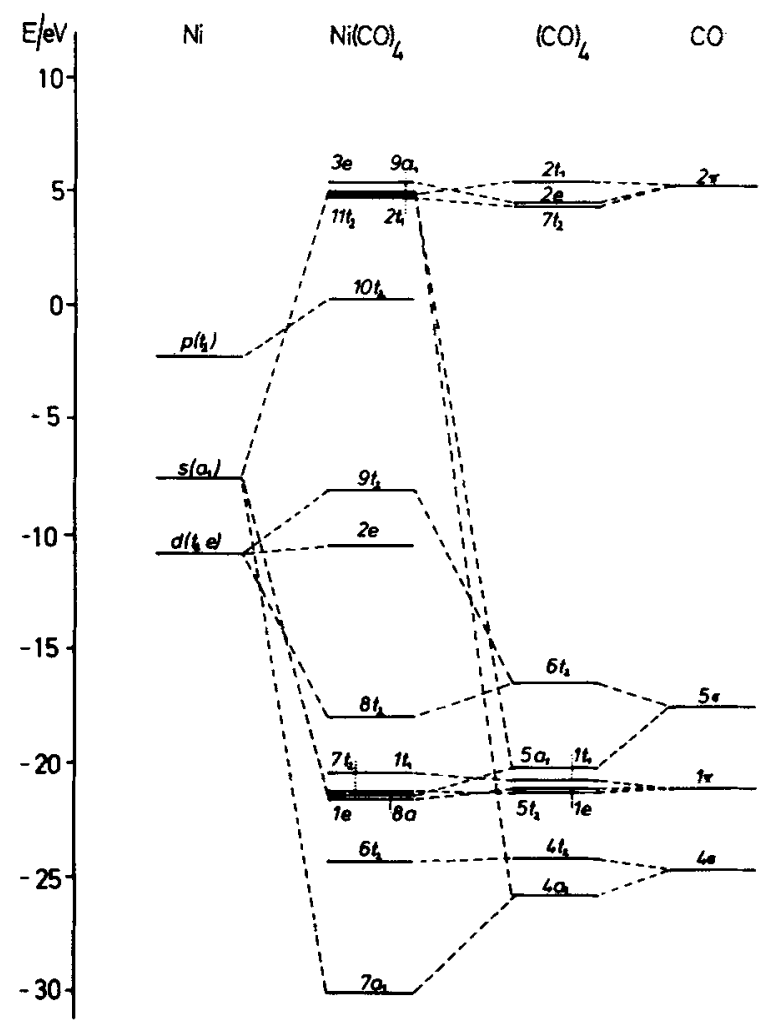

Figure 1. MO-diagram for $\mathrm{Ni}(\mathrm{CO})_{4}$. The correlation lines indicate the parentage from the ligand system (CO) and the metal atom. The numbering of the $\mathrm{Ni}(\mathrm{CO})_{4}$ orbitals includes five $a_{1}$ and four $t_{2}$ orbitals resulting from the inner shells. 
the metal atom. The two highest occupied orbitals $\left(2 e\right.$ and $\left.9 t_{2}\right)$ both have strong metal- $d$-character. The orbital energies compare quite well with the experimental IPs $\left(\mathrm{IP}_{1}=8.9 \mathrm{eV}, \mathrm{IP}_{2}=9.8 \mathrm{eV}\right)$ [14]. The lowest unoccupied orbital $10 t_{2}$ results mainly from the $4 p$ orbitals of the metal atom. About $4.5 \mathrm{eV}$ higher in energy are four closely spaced MOs: $9 a_{1}$ is primarily the Ni-4s orbital mixed with some contribution from $\mathrm{CO} 4 \sigma$ and $5 \sigma$. The three others $\left(11 t_{2}, 2 t_{1}\right.$ and $\left.3 e\right)$ originate from ligand $2 \pi$-orbitals. In contrast, in the EHT calculation by Schreiner and Brown [16] the low lying virtual orbitals did not contain significant metal $4 s$ and $4 p$ contributions, because of the large Slater exponents used for these AOs. The dominant orbital characters are used to label different transitions.

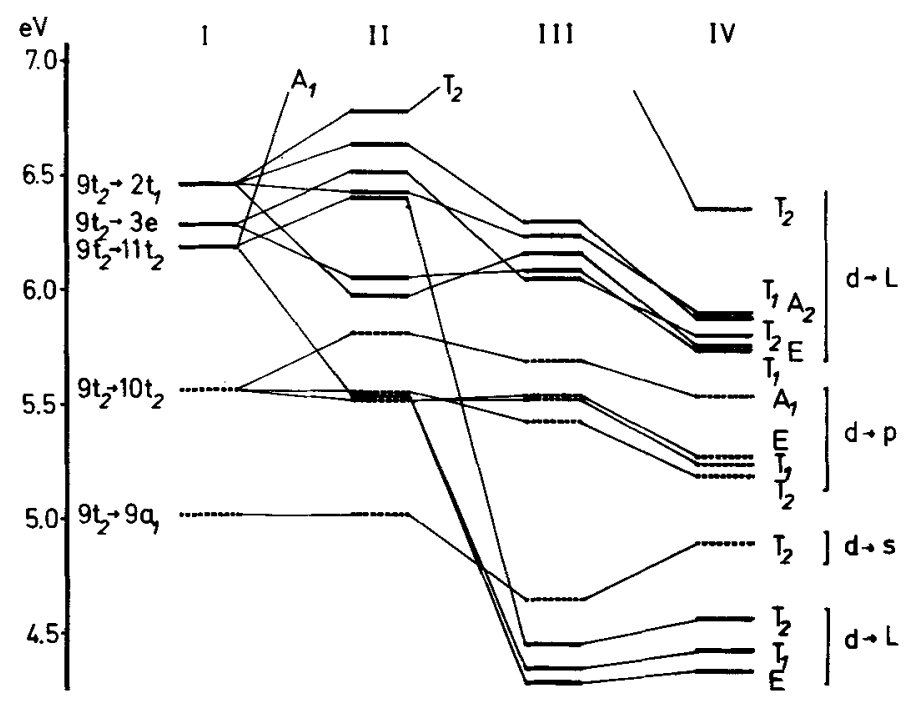

Fig. 2, Excited states of $\mathrm{Ni}(\mathrm{CO})_{4}$ as derived from the one particle picture and different CI-calculations (see text). The symmetry symbols at the left column indicate the orbital, to which excitation takes place from $9 t_{2}$.

In column I of figure 2 the lowest mean configuration energies are shown which result from the different one electron excitations. All excitations below $6.5 \mathrm{eV}$ start from the highest occupied orbital $9 t_{2}$, but the order is completely different from what we should expect from the orbital sequence. The atom like $d \rightarrow p$ transitions $9 t_{2} \rightarrow 10 t_{2}$ and $2 e \rightarrow 10 t_{2}$ no longer yield the lowest excitations, since the Coulomb integrals associated with these two transitions are small compared to those of the other transitions. Lowest order CI where only cross terms between configurations resulting from the same one electron excitation are included leads to the result shown in column II. While the zero order splitting of the $9 t_{2} \rightarrow 10 t_{2}$ transition is relatively small, the $d \rightarrow L$ transitions and in particular the $9 t_{2} \rightarrow 11 t_{2}$ transitions are split considerably.

The CI with all singly excited configurations leads to the results in column III. A first group of $d \rightarrow L$ transitions has been pushed below the $d \rightarrow s$ and the $d \rightarrow p$ transitions. A CI with 1200 energy selected singly and doubly excited configurations gave the result in the last column of figure 2 . At that level of 
approximation excited states are found in many organic molecules which contain 40 per cent and more DECs $[5,6]$. In $\mathrm{Ni}(\mathrm{CO})_{4}$, however, inclusion of DECs leads only to small changes in excitation energies (column IV) and to no important changes in the order of excited states. The contribution of DECs to the low lying excited states is less than 10 per cent for all states (table 2). This result is in line with an early suggestion of Koutecky [7] that correlation effects are less important in compact systems.

Table 2. Calculated excitation energies, oscillator strengths $(f)$ and two-photon absorption cross sections $(\delta)$ for $\mathrm{Ni}(\mathrm{CO})_{4}$. The ordering of the states refers to the SCI (full) calculation. Below the broken line not all states are given. The numbers in round brackets give the contribution of DECs (in per cent). $f_{1}$ and $\delta_{1}$ are calculated without and $f_{2}$ and $\delta_{2}$ with inclusion of the second term of (1). $\delta$-values are given in g.m. (Goeppert-Mayer) $=10^{-50} \mathrm{~cm}^{4} \mathrm{~s} / \mathrm{photon}$ molecule. For all $E$ and $T_{2}$ states $\Omega=1 \cdot 5$. n.c. $=$ not calculated.

\begin{tabular}{|c|c|c|c|c|c|c|c|c|}
\hline Symmetry & $\begin{array}{c}\text { Main } \\
\text { configuration }\end{array}$ & $\begin{array}{l}\text { Type of } \\
\text { excitation }\end{array}$ & $\begin{array}{l}\text { Excitatio } \\
\text { SCI (full) }\end{array}$ & $\begin{array}{l}\text { energy/eV } \\
\text { SDCI }(1200)\end{array}$ & $f_{1}$ & $f_{2}$ & $\delta_{1} / \mathrm{g} . \mathrm{m}$. & $\delta_{2} / \mathrm{g} \cdot \mathrm{m}$. \\
\hline $1 E$ & $9 t_{2} \rightarrow 11 t_{2}$ & $d \rightarrow L$ & $4 \cdot 27$ & $4 \cdot 32(5 \cdot 3)$ & - & - & 0.0005 & 1.7510 \\
\hline $1 T_{1}$ & $9 t_{2} \rightarrow 11 t_{2}$ & $d \rightarrow L$ & 4.32 & $4.41(5.2)$ & - & - & - & - \\
\hline $1 T_{2}$ & $9 t_{2} \rightarrow L$ & $d \rightarrow L$ & 4.44 & $4.55(5.4)$ & 0.0025 & 0.2294 & 0.0173 & $1 \cdot 3208$ \\
\hline $2 T_{2}$ & $9 t_{2} \rightarrow 9 a_{1}$ & $d \rightarrow s$ & 4.64 & $4.88(2.6)$ & 0.0174 & 0.0244 & 0.3323 & 0.3924 \\
\hline $3 T_{2}$ & $9 t_{2} \rightarrow 10 t_{2}$ & $d \rightarrow p$ & 5.42 & $5 \cdot 18(5 \cdot 8)$ & 0.0113 & 0.0007 & 0.0400 & 0.0310 \\
\hline $2 T_{1}$ & $9 t_{2} \rightarrow 10 t_{2}$ & $d \rightarrow p$ & 5.51 & $5 \cdot 23(6 \cdot 3)$ & - & - & - & - \\
\hline $2 E$ & $9 t_{2} \rightarrow 10 t_{2}$ & $d \rightarrow p$ & 5.52 & $5 \cdot 26(6 \cdot 1)$ & - & - & 0.1255 & 1.0507 \\
\hline $2 A_{1}$ & $9 t_{2} \rightarrow 10 t_{2}$ & $d \rightarrow p$ & 5.68 & $5.53(5.3)$ & - & - & n.c. & n.c. \\
\hline $4 T_{2}$ & $9 t_{2} \rightarrow 11 t_{2}$ & $d \rightarrow L$ & 6.04 & $5.79(7.1)$ & 0.0000 & 0.0018 & n.c. & n.c. \\
\hline $3 T_{1}$ & $9 t_{2} \rightarrow 3 e$ & $d \rightarrow L$ & 6.07 & $5.72(7.4)$ & - & - & - & - \\
\hline $3 E$ & $9 t_{2} \rightarrow 2 t_{1}$ & $d \rightarrow L$ & $6 \cdot 15$ & $5.73(7.8)$ & - & - & n.c. & n.c. \\
\hline $4 T_{1}$ & $9 t_{2} \rightarrow 2 t_{1}$ & $d \rightarrow L$ & $6 \cdot 22$ & $5.88(8.1)$ & - & - & - & - \\
\hline $1 A_{2}$ & $9 t_{2} \rightarrow 2 t_{1}$ & $d \rightarrow L$ & 6.29 & 5.86 & - & - & n.c. & n.c. \\
\hline $5 T_{2}$ & & & 7.76 & $6 \cdot 34$ & $0 \cdot 2490$ & 0.2149 & & \\
\hline
\end{tabular}

The oscillator strengths and two-photon cross sections given in table 2 are, for storage reasons, from a reduced SCI calculation neglecting the four highest virtual and the four lowest occupied orbitals. Since excitation energies are only slightly affected by this neglect, we expect the same for other one electron operators, too. In any case, the neglect of the third term in (1) is a much more serious restriction. Inclusion of one centre contributions (second term of (1) strongly increases the transition probability for the transition into $1 T_{2}$. For all other low lying one photon allowed transitions $1 A_{1} \rightarrow n T_{2}$ the transition moments are either small or only little affected by one centre contributions. The considerable intensity enhancement found for $1 A_{1} \rightarrow 1 T_{2}$ is due to a nonnegligible contribution of local ligand excitation of $\sigma \rightarrow \pi$ type, an excitation which in the framework of the CNDO approximation obtains all its intensity from one centre contributions. The fact that the order of magnitude is described quite well for free $\mathrm{CO}$ in this approximation makes it probable that the $f_{2}$-values are a reasonable guideline for the interpretation of the experimental 
spectrum. To gain information on the importance of the third term in (1) an investigation based on $a b$ initio calculations would be of interest.

The influence of the one centre term is even more pronounced for the twophoton cross sections $\delta$ than for the $f$-values. This had to be expected since only $T_{2}$-states can act as virtual intermediate states in the two-photon process and the only low lying $T_{2}$-state which exhibits a reasonable large $f_{2}$-value is $1 T_{2}$, the state for which the intensity results mainly from one-centre contributions. Thus two-photon allowed transitions are predicted to be very weak for transitions into $1 E, 1 T_{2}$ and $2 E$ if the second term in (1) is neglected but to be of moderate intensity (compared to other theoretical results [13]), if these terms are included. Only the $\delta$-values for the atom like $d \rightarrow s$ and $d \rightarrow p$ transitions into $2 T_{2}$ and $3 T_{2}$ change very little.

Unfortunately a comparison of our theoretical findings with experimental data is restricted by the fact that only low resolution absorption spectra are available [1 (b)]. In these spectra three bands can be discerned below $6.5 \mathrm{eV}$ $\left(52000 \mathrm{~cm}^{-1}\right)$ indicated by a maximum at about $6.0 \mathrm{eV}\left(\epsilon \sim 10^{5}\right)$ and two shoulders at about $5.5 \mathrm{eV}\left(\epsilon \sim 10^{4}\right)$ and $5.2 \mathrm{eV}\left(\epsilon \sim 10^{3}\right)$. All three bands have been assigned to $M \rightarrow L \mathrm{CT}$-transitions by Schreiner and Brown [1 (b)].

Basically our calculations yield excitation energies in the correct energy range (in [1 (b)] e.g. the calculated energy for the first transition was $2.68 \mathrm{eV}$ ). The assignment is, however, not quite clear; transitions to the final states $2 T_{2}, 3 T_{2}$ and $4 T_{2}$ are predicted to be weak. It is therefore very unlikely that one of these transitions is responsible for the intense band at about $6 \mathrm{eV}$. The first possible candidate assignable to this band is the metal to ligand CTtransition $1 A_{1} \rightarrow 5 T_{2}$ calculated at $7.3 \mathrm{eV}$ in SCI (full) and at 6.3 in SDCI $(1200)$. Since we have to include an approximate solvent shift of about $3000 \mathrm{~cm}^{-1}$ [5], this assignment is not unrealistic. Thus we are left with two bands in the low energy part of the spectrum. Since the $f_{2}$-values are more reliable the only possible assignment for the medium intense band at $5 \cdot 5 \mathrm{eV}$ is to a transition into $1 T_{2}$. Consequently, two assignments can be given for the weak band between 4.5 and $5.3 \mathrm{eV}$ :

(a) a transition into $1 E$ or $1 T_{1}$ which gains intensity via vibronic coupling ;

(b) the stabilization of the state labelled $1 T_{2}$ is overestimated in the SCI (full) and SDCI (1200) calculations and the $d \rightarrow s$ transition is still the lowest one.

A choice between these two assignments could be based on (i) high resolution spectroscopy (either in the gas phase or in solution at low temperature) since only in case $(b)$ it is possible to observe the $\mathrm{O}-\mathrm{O}$ transition of this band or (ii) a two-photon absorption spectrum since the $1 E$ state should lead to a medium intense band in this spectrum according to the calculated $\delta_{2}$-value.

\subsection{Photochemical behaviour of $\mathrm{Ni}(\mathrm{CO})_{4}$}

The exact nature of the active excited states is not clear. The only fact which seems to be supported experimentally [15] is that the primary step is indeed the cleavage of a single $\mathrm{Ni}-\mathrm{CO}$ bond

$$
\mathrm{Ni}(\mathrm{CO})_{4} \rightarrow \mathrm{Ni}(\mathrm{CO})_{3}+\mathrm{CO}
$$


Table 3. Charge density at the metal atom (relative to the grounds state value 9.62), Wiberg indices and changes in Mulliken overlap population for the low lying excited states of $\mathrm{Ni}(\mathrm{CO})_{4}$.

\begin{tabular}{|c|c|c|c|c|c|c|}
\hline Symmetry & $\begin{array}{l}\text { Type of } \\
\text { excitation }\end{array}$ & $E$ & $\Delta Q_{\mathrm{Ni}}$ & $W_{\mathrm{N} i-\mathrm{C}}$ & $W_{\text {C_o }}$ & $\Delta M_{\mathrm{Ni}-\mathrm{C}}$ \\
\hline $1 A_{1}$ & Ground state & 0.00 & 0.0000 & 0.5068 & $2 \cdot 3770$ & 0.0000 \\
\hline $1 E$ & $d \rightarrow L$ & $4 \cdot 27$ & $-0 \cdot 3462$ & 0.4825 & $2 \cdot 2351$ & 0.0296 \\
\hline $1 T_{1}$ & $d \rightarrow L$ & $4 \cdot 32$ & -0.3359 & 0.4708 & $2 \cdot 2424$ & 0.0242 \\
\hline $1 T_{2}$ & $d \rightarrow L$ & 4.44 & -0.3509 & 0.4778 & 2.2353 & 0.0268 \\
\hline $2 T_{2}$ & $d \rightarrow s$ & 4.64 & -0.0134 & 0.3742 & $2 \cdot 4028$ & -0.6353 \\
\hline $3 T_{2}$ & $d \rightarrow p$ & $5 \cdot 42$ & 0.0627 & 0.4890 & $2 \cdot 3586$ & 0.0262 \\
\hline $2 T_{1}$ & $d \rightarrow p$ & $5 \cdot 51$ & 0.1346 & 0.4915 & 2.3679 & 0.0283 \\
\hline $2 E$ & $d \rightarrow p$ & $5 \cdot 52$ & 0.1528 & 0.4978 & 2.3722 & 0.0319 \\
\hline $2 A_{1}$ & $d \rightarrow p$ & 5.68 & 0.3116 & 0.4887 & 2.4066 & 0.0307 \\
\hline $4 T_{2}$ & $d \rightarrow L$ & 6.04 & -0.3586 & 0.4618 & 2.2854 & 0.0139 \\
\hline $3 T_{1}$ & $d \rightarrow L$ & 6.07 & -0.4374 & 0.4571 & 2.2734 & 0.0118 \\
\hline $3 E$ & $d \rightarrow L$ & $6 \cdot 15$ & -0.4941 & 0.4771 & 2.2577 & 0.0204 \\
\hline $4 T_{1}$ & $d \rightarrow L$ & $6 \cdot 22$ & -0.6487 & 0.4816 & $2 \cdot 2292$ & 0.0227 \\
\hline $4 E$ & $d \rightarrow s$ & 6.58 & $-0 \cdot 1062$ & 0.3295 & 2.4119 & -0.6935 \\
\hline
\end{tabular}

Inspection of table 3, where we have collected charge densities, Wiberg indices [16] and differences of Mulliken overlap population [17] for the lowest electronic states suggests the following.

For all states which we have characterized as metal-to-ligand charge transfer states $(d \rightarrow L)$ we find at least a reduction of the electronic density at the central atom by $1 / 3$ of an electron. On the other hand an enhancement of the electron density at the central atom is found for all atom-like $d \rightarrow p$ excitations due to the bonding character of the metal $d$-orbitals and the localization of the $p$ orbitals.

The Wiberg indices for the metal-carbon bond are similar to the ground state value for all low lying excited states. Only for the quasi atomic $d \rightarrow s$ transitions a somewhat reduced value is found. The change in overlap population, however, is highly negative for these excitations, while it is close to zero for all others. This indicates that only the quasi atomic $d \rightarrow s$ excitations lead to excited states which are antibonding with respect to the metal ligand bond. We conclude therefore that it is this type of excitation which is responsible for the observed photo-dissociation. Unfortunately, no precise measurement of the wavelength dependence of the photo-dissociation can be found in the literature. The available information seems to indicate, however, that excitation in the first weak band is sufficient to produce photo-dissociation. This is a hint that assignment $(b)$ is the more likely one. It could be possible however, that photo-dissociation does not take part directly from the singlet [18] state but from the triplet state corresponding to $2^{1} T_{2}$. This triplet has similar bonding characteristics as $2^{1} T_{2}$ and is calculated to lie energetically close to $1^{1} T_{1}$ and $1^{\mathbf{l}} \mathrm{E}$. The measured lifetime of $7 \mathrm{~ns}$ [27] for the dissociative state makes it, unlikely, however, that a triplet is involved. A careful experimental study of 
the energy dependence of the photo-dissociation process is highly desired as detailed comparison of the absorption spectrum with the quantum yield of the photo reaction should allow us to decide between the different assignments.

\subsection{Excited states and U.V. spectrum of $\mathrm{Fe}(\mathrm{CO})_{5}$}

$\mathrm{Fe}(\mathrm{CO})_{5}$ provides an example where $d \rightarrow d$-transitions should occur in addition to those types of transitions found in $\mathrm{Ni}(\mathrm{CO})_{4}$. As usual these $d \rightarrow d$ transitions are expected to be among the lowest ones in energy and probably responsible for the weak shoulder found in the experimental spectrum at about $35000 \mathrm{~cm}^{-1}[1(\mathrm{c})]$.

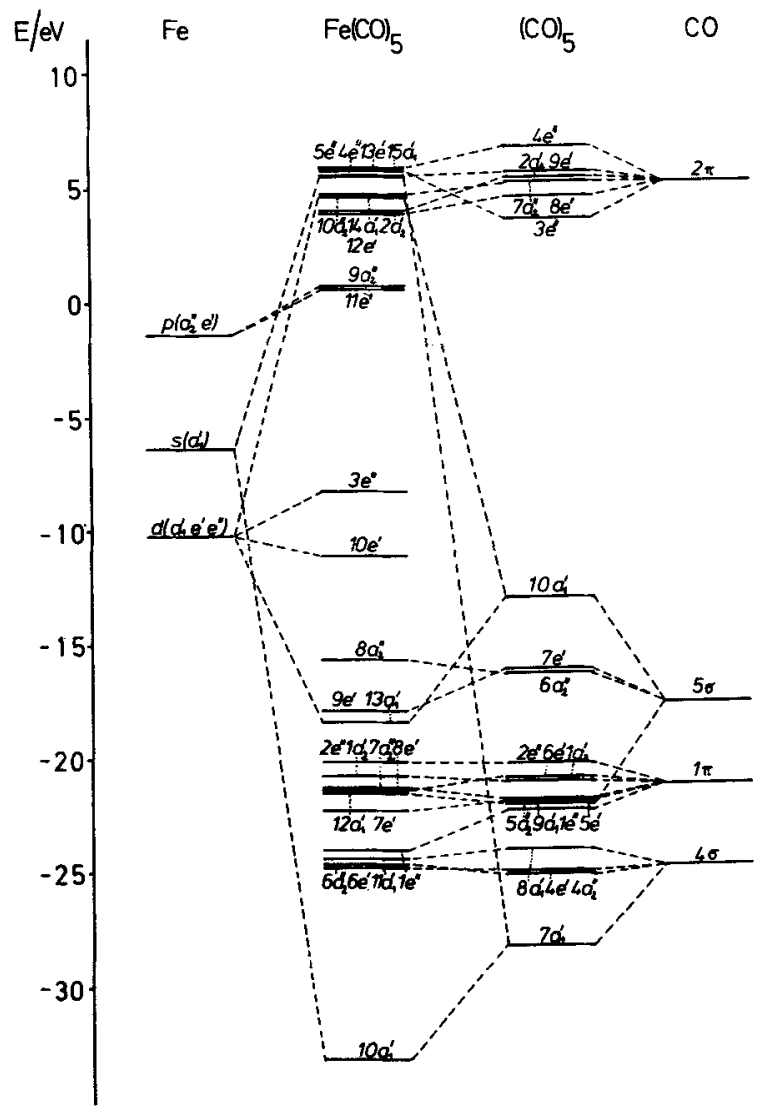

Figure 3. MO-diagram for $\mathrm{Fe}(\mathrm{CO})_{5}$. The correlation lines indicate the parentage from the ligand system $(\mathrm{CO})_{5}$ and the metal atom. The numbering of the $\mathrm{Fe}(\mathrm{CO})_{5}$ orbitals includes seven $a_{1}$, four $e^{\prime}$ and four $a_{2}^{\prime \prime}$ orbitals from the inner shells.

The calculated orbital energies are shown in figure 3 in the same fashion as for $\mathrm{Ni}(\mathrm{CO})_{4}$. The two highest occupied orbitals $\left(10 e^{\prime}\right.$ and $\left.3 e^{\prime \prime}\right)$ are well separated from the lower ones in complete accord with the photoelectron spectrum $\left(\mathrm{IP}_{1}=8.6 \mathrm{eV}, \mathrm{IP}_{2}=9.9 \mathrm{eV}\right)[19]$. Both have pronounced $d$-character but not as strong as the corresponding orbitals in $\mathrm{Ni}(\mathrm{CO})_{4}$. The set of unoccupied 
MOs separates into two groups, one having energies between 0.67 and $5.84 \mathrm{eV}$ and the other above $13 \mathrm{eV}$. The two lowest unoccupied orbitals have nearly pure metal $p$-character. The largest $s$-contribution is found in $15 a_{1}^{\prime}$ (52 per cent) and the largest $d$-contribution in $14 a_{1}^{\prime}$ ( 40 per cent) and $5 e^{\prime \prime}$ ( 25 per cent).

The order of the mean configuration energies (figure 4 , column I) again differs considerably from the order of the orbital energy differences. All low lying excitations result from transitions starting from the orbital $10 e^{\prime}$. We indicate therefore only the final orbital in figure 4 . The lowest excitation is now the $d \rightarrow d$ transition $10 e^{\prime} \rightarrow 14 a^{\prime}{ }_{1}$ in accord with an assignment derived by Dartiguenave et al. $[1(c)]$ from EHT (our $14 a_{1}^{\prime}$ is $5 a_{1}^{\prime}$ in their notation).

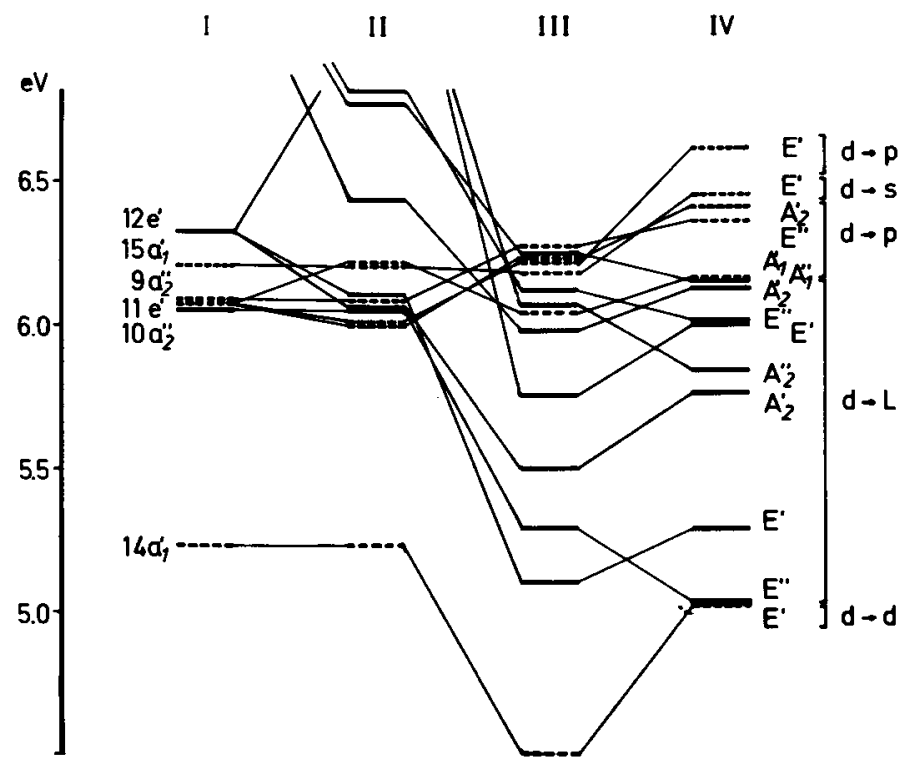

Figure 4. Excited states of $\mathrm{Fe}(\mathrm{CO})_{5}$ as derived from the one particle picture and different CI-calculations (see text). The symmetry symbols at the left column indicate the orbital, to which excitation takes place from $10 e^{\prime}$.

Inclusion of $\mathrm{CI}$ among degenerate configurations leads to the result shown in column II. Again the zero order splitting is only small for the atom like $d \rightarrow p$ transition $10 e^{\prime} \rightarrow 11 e^{\prime}$ but very strong for the $d \rightarrow L$ transition $10 e^{\prime} \rightarrow 12 e^{\prime}$. Further extension of the CI to all singly excited configurations (column III) results in a considerable stabilization of all low lying excited states except those with predominant $d \rightarrow s$ or $d \rightarrow p$ character. This is again in line with what we have found for $\mathrm{Ni}(\mathrm{CO})_{4}$. By inclusion of doubly excited configurations most of the excited states are stabilized less than the ground state (column IV). Only a few are stabilized more $\left(1 E^{\prime \prime}, 1 A^{\prime \prime}{ }_{2}, 2 E\right.$ and $\left.2 A^{\prime \prime}{ }_{2}\right)$, a behaviour known from the covalent states in hydrocarbons [6]. The different stabilizations lead to a reordering of the lowest excited states $; 1 E^{\prime \prime}$ is now found very close to $1 E^{\prime}$.

To calculate oscillator strengths and two-photon cross sections we used a SCI [15 $\times 19]$ calculation including all configurations with diagonal elements smaller than $1 E_{\mathrm{h}}$. The energies obtained with this calculation differ by less than $0.2 \mathrm{eV}$ from the SCI (full) result. Only the values obtained with inclusion 
of one-centre terms (second term of (1)) are shown in table 4. One photon transitions are allowed to final states of symmetry $E^{\prime}$ and $A^{\prime \prime}{ }_{2}$ and two-photon transitions to final states of symmetry $E^{\prime}, E^{\prime \prime}$ and $A^{\prime}{ }_{1}$. To our knowledge measurements of the two-photon absorption cross section have not yet been reported for $\mathrm{Fe}(\mathrm{CO})_{5}$. The one-photon absorption spectrum [1(c)] shows a maximum $(\epsilon \sim 37000)$ around $6.3 \mathrm{eV}$, an indication of a shoulder at about $5 \cdot 8 \mathrm{eV}$ and two clearly discernible shoulders around $5 \cdot 2(\epsilon \sim 10000)$ and $4.4 \mathrm{eV}$ $(\epsilon \sim 4000)$. If this is compared with our calculated energies it is found that the onset of the spectrum is described quite well, especially by the SCI (full) calculation. The first weak shoulder results most likely from the transition $1 A^{\prime}{ }_{1} \rightarrow 1 E^{\prime}$, a transition with predominant $d \rightarrow d$-character but some admixtures of $d \rightarrow s, d \rightarrow L$ and $L \rightarrow L$.

Table 4. Calculated excitation energies, oscillator strengths $(f)$ and two-photon absorption cross sections $(\delta)$ for $\mathrm{Fe}(\mathrm{CO})_{5}$. The same nomenclature is used as in table 2. For the main configurations only the final orbitals are given since all excitations originate from 10 $e^{\prime}$. The number in brackets gives the occupation (in per cent).

\begin{tabular}{|c|c|c|c|c|c|c|c|}
\hline Symmetry & $\begin{array}{l}\text { Main configuration } \\
\text { SCI (full) }\end{array}$ & $\begin{array}{l}\text { Type of } \\
\text { excitation }\end{array}$ & $\begin{array}{l}\text { Excitation } \\
\text { SCI (full) }\end{array}$ & $\begin{array}{l}\text { energy/eV } \\
\text { SDCI }(1200)\end{array}$ & $f_{2}$ & $\delta_{2}$ & $\Omega$ \\
\hline $1 E^{\prime}$ & $14 a_{1}^{\prime}(84)$ & $d \rightarrow d$ & $4 \cdot 50$ & $5.02(4.1)$ & 0.0008 & 0.0905 & 1.50 \\
\hline $2 E^{\prime}$ & $2 a_{1}^{\prime}(63), 13 e^{\prime}(27)$ & $d \rightarrow L$ & $5 \cdot 10$ & $5 \cdot 29(4 \cdot 6)$ & 0.0009 & 0.3003 & 1.50 \\
\hline $1 E^{\prime \prime}$ & $10 a_{2}^{\prime}(76)$ & $d \rightarrow L$ & $5 \cdot 29$ & $5.03(7.6)$ & - & $0 \cdot 1506$ & 1.50 \\
\hline $1 A_{2}^{\prime}$ & $11 e^{\prime}(30), 12 e^{\prime}(36), 13 e^{\prime}(32)$ & $d \rightarrow L$ & $5 \cdot 50$ & $5 \cdot 76(4 \cdot 5)$ & - & 一 & - \\
\hline $3 E^{\prime}$ & $11 e^{\prime}(36), 12 e^{\prime}(53)$ & $d \rightarrow L$ & $5 \cdot 75$ & $6.00(3.7)$ & 0.0079 & $0 \cdot 1151$ & 1.50 \\
\hline $2 A_{2}^{\prime}$ & $12 e^{\prime}(30), 13 e^{\prime}(61)$ & $d \rightarrow L$ & 5.98 & $6 \cdot 12(5 \cdot 8)$ & - & - & - \\
\hline $2 A_{1}^{\prime}$ & $11 e^{\prime}(82)$ & $d \rightarrow p$ & $6 \cdot 04$ & $6 \cdot 16(4.7)$ & - & 0.0300 & $0 \cdot 28$ \\
\hline $1 A^{\prime \prime}{ }_{2}$ & $4 e^{\prime \prime}(36), 5 e^{\prime \prime}(60)$ & $d \rightarrow L$ & 6.07 & $5.84(8.2)$ & 0.0726 & - & - \\
\hline $2 E^{\prime \prime}$ & $9 a^{\prime \prime}{ }_{2}(21), 4 e^{\prime \prime}(22), 5 e^{\prime \prime}(53)$ & $d \rightarrow L$ & $6 \cdot 12$ & $6 \cdot 01(8 \cdot 1)$ & - & 0.3904 & $1 \cdot 50$ \\
\hline $4 E^{\prime}$ & $15 a_{1}^{\prime}(66)$ & $d \rightarrow s$ & $6 \cdot 18$ & $6.45(3.6)$ & 0.0229 & 0.0877 & $1 \cdot 50$ \\
\hline $5 E^{\prime}$ & $11 e^{\prime}(56), 12 e^{\prime}(28)$ & $d \rightarrow p$ & $6 \cdot 22$ & $6 \cdot 61(4 \cdot 1)$ & 0.0009 & 0.0574 & 1.50 \\
\hline $3 A_{2}^{\prime}$ & $11 e^{\prime}(62), 12 e^{\prime}(30)$ & $d \rightarrow p$ & $6 \cdot 22$ & $6.41(3.9)$ & - & - & 一 \\
\hline $1 A_{1}^{\prime \prime}$ & $5 e^{\prime \prime}(73)$ & $d \rightarrow L$ & $6 \cdot 25$ & $6 \cdot 16(9 \cdot 0)$ & - & - & - \\
\hline $3 E^{\prime \prime}$ & $9 a_{2}^{\prime \prime}(62)$ & $d \rightarrow p$ & $6 \cdot 27$ & $6 \cdot 36(4 \cdot 7)$ & - & n.c. & $1 \cdot 50$ \\
\hline $3 A_{1}^{\prime}$ & $12 e^{\prime}(72)$ & $d \rightarrow L$ & 6.53 & $6.58(4.5)$ & - & n.c. & n.c. \\
\hline $4 E^{\prime \prime}$ & $12 e^{\prime}(43), 4 e^{\prime \prime}(40)$ & $d \rightarrow L$ & 6.88 & $7 \cdot 47(3 \cdot 2)$ & - & n.c. & 1.50 \\
\hline $6 E^{\prime}$ & & & $7 \cdot 10$ & 7.05 & 0.4147 & n.c. & 1.50 \\
\hline
\end{tabular}

The first intense transition assignable to the band observed around $6 \cdot 3 \mathrm{eV}$ is $1 A^{\prime}{ }_{1} \rightarrow 6 E^{\prime}$ calculated at $7 \cdot 10 \mathrm{eV}$ in $\mathrm{SCI}$ (full) and $7 \cdot 05 \mathrm{eV}$ in $\mathrm{SDCI}(1200)$. The energy is somewhat overestimated in the calculations, even if we take into account the usual estimation for the solvent shift. The assignment of the band around $5 \cdot 2 \mathrm{eV}$ is uncertain. $2 E^{\prime}$ which would fit very well in energy has an $f$-value much too low to be responsible for a transition with $\epsilon \sim 10000$. The only reasonable candidates are $1 A^{\prime}{ }_{1} \rightarrow 4 E^{\prime}$ and $1 A^{\prime}{ }_{1} \rightarrow 1 A^{\prime \prime}{ }_{2}$. Most likely both transitions contribute to the fairly broad band. It is possible, however, that the 
band results from only one of these transitions while the other is responsible for the weakly indicated shoulder at about $5.8 \mathrm{eV}$. Two-photon spectroscopy could resolve this question since $1 A^{\prime}{ }_{1} \rightarrow 1 A^{\prime \prime}{ }_{2}$ is two-photon forbidden.

\subsection{Photochemical behaviour of $\mathrm{Fe}(\mathrm{CO})_{5}$}

Charge densities, Wiberg indices and differences of Mulliken overlap population obtained from the SCI (full) calculation are collected in table 5. In the ground state the iron atom is slightly positive $(0.49)$ and the Wiberg indices indicate a somewhat stronger bonding for the axial than for the equatorial ligands. The same is also true for all low lying excited states.

Table 5. Charge density at the metal atom (relative to the ground state value 7.51 ), Wiberg indices and changes in Mulliken overlap population for the low lying excited states of $\mathrm{Fe}(\mathrm{CO})_{5}$.

\begin{tabular}{|c|c|c|c|c|c|c|c|c|}
\hline Symmetry & $E$ & $\Delta Q_{\mathrm{Fe}}$ & $W_{\mathrm{Fe}-\mathrm{C}^{\mathrm{ax}}}$ & $W_{\mathrm{Fe}-\mathrm{C}}^{\mathrm{eq}}$ & $W_{\mathrm{CO}^{\mathbf{a x}}}$ & $W_{\mathrm{Co}^{\mathrm{eq}}}$ & $\Delta M_{\mathrm{Fe}} \mathrm{c}^{\mathrm{ax}}$ & $\Delta M_{\mathrm{Fec}}{ }^{\mathrm{eq}}$ \\
\hline $1 A_{1}^{\prime}$ & 0.00 & 0.0000 & 0.7975 & 0.7134 & 2.2511 & $2 \cdot 2560$ & 0.0000 & 0.0000 \\
\hline $1 E^{\prime}$ & $4 \cdot 50$ & $-0 \cdot 0161$ & 0.6674 & 0.5674 & $2 \cdot 2563$ & $2 \cdot 2946$ & $-0 \cdot 1171$ & -0.1856 \\
\hline $2 E^{\prime}$ & $5 \cdot 10$ & -0.2824 & 0.7787 & 0.6812 & 2.2596 & $2 \cdot 1149$ & -0.0093 & 0.0271 \\
\hline $1 E^{\prime \prime}$ & $5 \cdot 29$ & -0.3037 & 0.7818 & 0.7533 & $2 \cdot 2681$ & 2.0980 & -0.0064 & 0.0592 \\
\hline $1 A_{2}^{\prime}$ & $5 \cdot 50$ & -0.1522 & 0.8017 & 0.6355 & $2 \cdot 1443$ & $2 \cdot 2442$ & 0.0080 & -0.0056 \\
\hline $3 E^{\prime}$ & 5.75 & -0.1989 & 0.8229 & 0.6776 & 2.0611 & 2.2936 & -0.0242 & $0 \cdot 0336$ \\
\hline $2 A_{2}^{\prime}$ & 5.98 & -0.3225 & 0.8109 & 0.6442 & $2 \cdot 1358$ & $2 \cdot 1805$ & +0.0023 & 0.0238 \\
\hline $2 A_{1}^{\prime}$ & 6.04 & +0.2582 & 0.8071 & 0.6730 & $2 \cdot 2112$ & $2 \cdot 3001$ & 0.0061 & 0.0274 \\
\hline $1 A_{2}^{\prime \prime}$ & 6.07 & -0.2238 & 0.7722 & 0.6340 & 2.2672 & 2.0938 & -0.0098 & 0.0129 \\
\hline $2 E^{\prime \prime}$ & $6 \cdot 12$ & -0.1273 & 0.7633 & 0.6545 & $2 \cdot 2601$ & $2 \cdot 1460$ & -0.0142 & 0.0153 \\
\hline $4 E^{\prime}$ & $6 \cdot 18$ & +0.0705 & 0.6641 & 0.6506 & $2 \cdot 2166$ & $2 \cdot 2969$ & -0.8783 & -0.3626 \\
\hline $5 E^{\prime}$ & $6 \cdot 22$ & +0.1887 & 0.7690 & 0.6878 & $2 \cdot 1932$ & $2 \cdot 3084$ & -0.2242 & -0.0531 \\
\hline $3 A_{2}^{\prime}$ & $6 \cdot 22$ & +0.1924 & 0.7965 & 0.6988 & $2 \cdot 1869$ & $2 \cdot 3055$ & -0.0732 & -0.0216 \\
\hline $3 E^{\prime \prime}$ & $6 \cdot 27$ & +0.1545 & 0.7778 & 0.6997 & $2 \cdot 2600$ & $2 \cdot 2404$ & -0.0042 & 0.0305 \\
\hline
\end{tabular}

Significant changes $\Delta M_{\mathrm{Fec}}$ are only found for the quasi-atomic transitions leading to the states $1 E^{\prime}(d \rightarrow d), 4 E^{\prime}(d \rightarrow p)$ and $5 E^{\prime}(d \rightarrow s)$, indicating that the carbon-iron bond is at least weakened in these excited states. For $1 E^{\prime}$ the absolute value of $\Delta M_{\mathrm{Fec}}$ is somewhat larger for the equatorial than the axial position, but both values are considerably smaller than that found for the $2 T_{2}$-state of $\mathrm{Ni}(\mathrm{CO})_{4}$. From this we should conclude that the first excited state is the state relevant for the photochemical decomposition of $\mathrm{Fe}(\mathrm{CO})_{5}$ and that an equatorial ligand is released with higher probability than an axial one.

Experimentally the photochemical activity of $\mathrm{Fe}(\mathrm{CO})_{5}$ is well known [20], indicating that a dissociative low lying state must indeed exist. Photosubstitution reactions lead to substitution, both in equatorial [21] and axial positions [22], depending on the nature of the substituting ligand. This is readily understood if we take into account the possibility of pseudorotation [23]. 
If the absolute value of $\Delta M_{\mathrm{FeC}}$ is a measure for the weakening of the bond, the photo-dissociation should be less efficient in $\mathrm{Fe}(\mathrm{CO})_{5}$ than in $\mathrm{Ni}(\mathrm{CO})_{4}$. Unfortunately, no relevant experimental data are found in the literature. It would also be interesting to know whether there is an increase in the yield of the photo-dissociation if the excitation reaches $4 E^{\prime}$. This transition has been assigned either to the shoulder observed around $5.2 \mathrm{eV}$ or to the weakly indicated shoulder at about $5 \cdot 8 \mathrm{eV}$. If $4 E^{\prime}$ is in fact strongly dissociative this should help to clarify the assignment.

\section{Conclusion}

From our present investigation of $\mathrm{Ni}(\mathrm{CO})_{4}$ and $\mathrm{Fe}(\mathrm{CO})_{5}$ a number of results have emerged which we expect to hold also for other simple carbonyls.

(i) An assignment of the U.V. spectrum based only on orbital energy differences as found in most of the earlier work in this field is not appropriate.

(ii) Excitations to metal $s$ - and $p$-orbitals should be considered in addition to $d \rightarrow d, M \rightarrow L$ charge transfer and local ligand excitations since excited states resulting mainly from the first type of excitations are predicted to be in the low energy range. Apart from possible $d \rightarrow d$ transitions the features observed in the low energy part of the one-photon absorption spectrum result, however, mainly from metal to ligand CT-type excitations. So far some of the earlier assignments are confirmed by this investigation.

(iii) The strong photochemical activity observed for most of the simple carbonyls is predicted to be connected with excited states which evolve from the atom like $d \rightarrow s$ and $d \rightarrow p$ excitations. More detailed experimental studies are necessary to test this prediction.

(iv) Specific correlation effects which result from the inclusion of doubly excited configurations are found to be less important than in organic molecules. This might be due to the relative compactness of the carbonyls.

We thank the 'Deutsche Forschungsgemeinschaft ' for financial support, the 'Rechenzentrum der Universität zu Köln ' for providing the necessary computer time and Dr. E. Weltin for many valuable discussions.

\section{REFERENCES}

[1] (a) Beach, N. A., and Gray, H. B., 1968, 9. Am. chem. Soc., 90, 5713. (b) Schreiner, A. F., and Brown, T. C., 1968, 7. Am. chem. Soc., 90, 3366. (c) Dartiguenave, M., Dartiguenave, Y., and Gray, H. B., 1969, Bull. Soc. chim. Fr., 4223. (d) Roebber, J. L., Wiener, R. N., and Russel, C. A., 1974, F. chem. Phys., 60, 3166. (e) Bratshko, Yu. I., and Nazarenko, Yu. P., 1974, Teor. Eksp. Khim., 10, 36. (f) Stoklosa, H. J., Wasson, J. R., and McCormick, B. J., 1974, Inorg. Chem., 13, 592.

[2] (a) Klotzbücher, W., Ozin, G. A., Norman, J. G., JR., and Kolari, H. J., 1977, Inorg. Chem., 16, 2871. (b) Weber, J., Geoffroy, M., Goursot, A., and Peningault, E., 1978, \%. Am. chem. Soc., 100, 3995. 
[3] (a) Freund, H.-J., and Hohlneicher, G., 1979, Theor. chim. Acta, 51, 145. (b) Freund, H.-J., Dick, B., and Hohlneicher, G., 1980, Theor. chim. Acta, 57, 181. (c) Saddei, D., Freund, H.-J., and Hohlneicher, G., 1980, Ғ. organomet. Chem. 186, 63. (d) Saddei, D., Freund, H.-J., and Hohlneicher, G., 1981, Chem. Phys., 55, 339.

[4] (a) Saddei, D., Freund, H.-J., and Hohlneicher, G., 1980, Surf. Sci., 95, 527. (b) Saddei, D., Freund, H.-J., and Hohlneicher, G., 1981, Surf. Sci., 102, 359.

[5] Dick, B., and Hohlneicher, G., 1980, Theor. chim. Acta, 53, 221.

[6] Schulten, K., Ohmine, I., and Karplus, M., 1976, J. chem. Phys., 64, 4422.

[7] Koutecky, J., 1967, J. chem. Phys., 46, 1501.

[8] (a) Pople, J. A., and Segal, G. A., 1965, F. chem. Phys., 43, 5136. (b) Pople, J. A., Santry, D. P., and Segal, G. A., 1965, Y. chem. Phys., 43, 8129. (c) Pople, J. A., and Segal, G. A., 1965, अ. chem. Phys., 44, 3289. (d) Pople, J. A., Beveridge, D. L., and Dobosh, P. A., 1967, f. chem. Phys., 47, 2026.

[9] (a) Clark, P. A., 1971, Y. chem. Phys., 54, 45. (b) Giessner-Prettre, C., and Pullman, A., 1969, Theor. chim. Acta, 13, $265 ;$ 1970, Theor. chim. Acta, 17, 120.

[10] Rank, A., and Barriel, J. M., 1977, Chem. Phys., 25, 409.

[11] (a) Ellis, R. L., Kuehnlenz, G., and Jaffé, H. H., 1972, Theor. chim. Acta, 26, 131. (b) Ellis, R. L., and JAFFÉ, H. H., 1977, Modern Theoretical Chemistry, edited by G. A. Segal, Vol. 8B (Plenum Press), p. 72.

[12] (a) Monson, P. R., and McClain, W. M., 1970, J. chem. Phys., 53, 29. (b) Monson, P. R., and McClain, W. M., 1972, Ұ. chem. Phys., 56, 4817.

[13] Hohlneicher, G., and Dick, B., 1979, Э. chem. Phys., 70, 5427.

[14] Hillier, J. H., Guest, M. F., Higginson, B. R., and Lloyd, D. R., 1974, Molec. Phys., 27, 215.

[15] Garratt, A. P., and Thompson, H. W., 1934, f. chem. Soc., 1817.

[16] Wiberg, K., 1968, Tetrahedron, 24, 1083.

[17] Mulliken, R. S., 1955, Ұ. chem. Phys., 23, 1833.

[18] MiснL, J., 1977, Modern Theoretical Chemistry, edited by G. A. Segal, Vol. 8B (Plenum Press).

[19] Lloyd, D. R., and Schlag, E. W., 1969, Inorg. Chem., 8, 2544.

[20] Thompson, H. W., and GarratT, A. P., 1934, J. chem. Soc., 524.

[21] Kettle, S. F. A., and Orgel, L. W., 1960, Chemy Ind., p. 49.

[22] Schubert, E. H., and Sheline, R. K., 1966, Inorg. Chem., 5, 1071.

[23] Cotton, A. F., and Wilkinson, G., 1978, Advanced Inorganic Chemistry (Verlag Chemie, Weinheim/Bergstraße).

[24] Lassettre, E. N., and Skerbele, A., 1971, J. chem. Phys., 54, 1597.

[25] Herzerrg, G., Hugo, T., Tilford, S., and Simmons, J., 1970, Can. Y. Phys., 48, 3004.

[26] Herzberg, G., 1939, Spectra of Diatomic Molecules (Van Nostrand).

[27] Callear, A. B., 1961, Proc. R. Soc. A, 265, 71. 